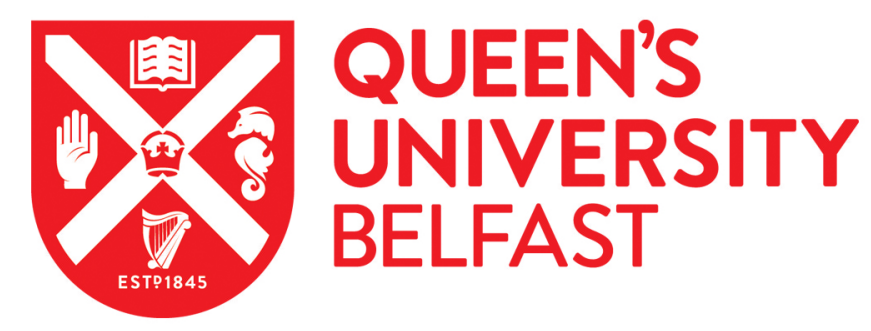

\title{
CVR and Loss Optimization through Active Voltage Management: A Trade-off Analysis
}

Gharavi Ahangar, H., Ochoa, L. F., Liu, X. A., Paterson, G., Ingham, B., \& McLoone, S. (2020). CVR and Loss Optimization through Active Voltage Management: A Trade-off Analysis. leee Transactions On Power Delivery. https://doi.org/10.1109/TPWRD.2020.3043245

Published in:

leee Transactions On Power Delivery

Document Version:

Peer reviewed version

Queen's University Belfast - Research Portal:

Link to publication record in Queen's University Belfast Research Portal

Publisher rights

Copyright 2020 IEEE

This work is made available online in accordance with the publisher's policies. Please refer to any applicable terms of use of the publisher.

\section{General rights}

Copyright for the publications made accessible via the Queen's University Belfast Research Portal is retained by the author(s) and / or other copyright owners and it is a condition of accessing these publications that users recognise and abide by the legal requirements associated with these rights.

Take down policy

The Research Portal is Queen's institutional repository that provides access to Queen's research output. Every effort has been made to ensure that content in the Research Portal does not infringe any person's rights, or applicable UK laws. If you discover content in the Research Portal that you believe breaches copyright or violates any law, please contact openaccess@qub.ac.uk. 


\title{
CVR and Loss Optimization through Active Voltage Management: A Trade-off Analysis
}

\author{
Hani Gharavi, Luis F. Ochoa, Senior Member, IEEE, Xueqin Liu, Geraldine Paterson, Ben Ingham, \\ Seán McLoone, Senior Member, IEEE
}

\begin{abstract}
Distribution companies are increasingly turning to active network management to improve how networks perform with respect to different metrics or objectives. This includes Conservation Voltage Reduction (CVR) that seeks to minimize energy usage of consumers (mostly connected to low voltage, $\mathrm{LV}$, levels) by reducing their supply voltage. However, if medium voltage (MV) levels have to be reduced to achieve CVR, it can negatively affect the energy losses in MV networks. In this study, we explore the potential trade-off that may exist between reducing energy consumption of $L V$ networks and the potential increase in energy losses in MV networks. A Pareto Particle Swarm Optimization (PPSO) is used to actively control voltage devices, including on-load tap changer-fitted $L V$ transformers and MV capacitors. Using three realistically modelled radially operated UK MV/LV networks serving $37,000+$ customers $(94 \%$ residential, $6 \%$ commercial and industrial), our results show that a trade-off does exist, but it can be reduced with increasing network control flexibility, particularly with controllable $L V$ transformers. Furthermore, for these networks the reduction in energy consumption due to CVR can be 20 times larger than the increase seen in MV energy losses. These insights are useful for distribution companies in assessing the overall benefits of CVR with active voltage management despite small increases in MV energy losses.
\end{abstract}

Index Terms- Energy Loss Reduction, Active Power Minimization, Multi-Objective Trade-off Optimization, PPSO.

\section{INTRODUCTION}

$\mathrm{C}$ ONSERVATION voltage reduction (CVR) is a network management strategy whereby the supply voltage to consumers is lowered to reduce their energy consumption. The move towards electrification of heating and transport is leading to a substantial increase in the electricity demand experienced by distribution networks. Due to the high cost associated with installing new infrastructure to meet this rising demand, active management through CVR is increasingly being considered as a means of reducing the total demand, and also as a means of creating additional capacity to accommodate low carbon technologies such as roof-top photovoltaic systems.

The history of CVR dates back to 1960 when it was first employed in the US to reduce demand in the face of power shortages [1], [2], [3] and [4]. Since then, it has been successfully demonstrated in trials and studies across the

Hani Gharavi, Xueqin Liu and Seán McLoone are with Queen's University Belfast, Belfast, Northern Ireland, BT29 5AH, UK (e-mail: hgharaviahangar01@qub.ac.uk,x.liu@qub.ac.uk and s.mcloone@qub.ac.uk). Luis F. Ochoa is with The University of Melbourne, Melbourne, Parkville VIC 3010, Australia and The University of Manchester, UK (e-mail: luis ochoa@ieee.org). Geraldine Paterson and Ben Ingham are with the Electricity North West Limited, Manchester, UK (e-mail: geraldine.paterson@enwl.co.uk and ben.ingham@enwl.co.uk).

This work was partly funded by Ofgem's Low Carbon Networks (LCN) Fund via Electricity North West Limited (ENWL), UK (Smart Street project, http://www.enwl.co.uk/smartstreet) and by a British Council Newton Institutional Links Programme grant with Helwan University, Egypt. globe, from open-loop implementations in the early days [5], [6], [7], [8], [9] to closed-loop implementations in the last decade [10], [11], [12]. CVR has been introduced to electrical networks using various approaches. Conventional open-loop CVR has been achieved using techniques such as line drop compensation, load tap changes, capacitor-based reduction, voltage spread reduction and home voltage reduction [3]. The closed-loop application of CVR through Voltage/Var control (VVC) has become viable with the role out of advanced metering infrastructure (AMI) and SCADA systems on distribution networks [3]. These monitoring systems allow distribution companies to monitor their networks in real-time and adapt operating conditions to optimize performance with respect to both short-term and long-term performance metrics. The outcome of using AMI based CVR systems in three different areas in the US was an $0.92 \%$ reduction in energy consumed for each $1 \%$ reduction in voltage [13].

Reducing energy consumption and minimizing power system losses have been the subjects of many investigations over several decades [14], [15] and [16]. The use of VVC to minimize medium voltage (MV) losses on distribution networks has also been investigated by a number of studies globally ([17], [18], [19]) with voltage control achieved using network control devices such as switchable capacitors, on-load tap changer (OLTC) transformers and tie switches for meshing and with monitoring provided by AMI and SCADA systems. For example, quadratic programming was used to minimize total energy loss through the installation and scheduled switching of static capacitors in a step by step iterative procedure in [14]. A centralized, three phases AC OPF-based CVR scheme was implemented as a mixed integer nonlinear program in [20] to minimize the overall energy consumption of a PV-rich unbalanced MV-low voltage (LV) distribution network in the UK. A Pareto-based multi-objective optimization using discrete particle swarm optimization (PSO) was used to simultaneously optimize power loss and the number of switching operations and deviations of bus voltages from their rated values subject to system constraints in [21].

Recently, Electricity North West Limited (ENWL) in the UK have conducting trials on actively managing voltage simultaneously across the MV and LV networks in order to reduce power consumption on the LV networks through CVR The trials were undertaken as part of an active management project called 'Smart Street' [22]. For a given MV voltage level, MV power losses will decrease due to reduced power flows arising from a reduction in energy consumption by the LV networks. However, infrastructure constraints with regard to changing LV transformer voltage levels may require reductions in the MV network voltage level in order to deliver the desired LV voltage reductions, thereby increasing MV losses. Hence, a trade-off may exist between energy losses on the MV side and CVR on the LV side of the distribution 
network. However, such a trade-off has not been fully investigated in the literature to date.

In this paper, we extend the work in [23] (a preliminary study restricted to a single $\mathrm{MV} / \mathrm{LV}$ network and load scenario), with a broader and more comprehensive investigation considering different types of distribution networks and operating scenarios, thereby providing a rigorous assessment for distribution companies to understand the extent of the trade-off that exists between the MV and LV network objectives when performing CVR with active voltage management. The study is conducted using detailed threephase simulations of three coupled MV/LV networks from the Smart Street trial networks, representative of dense urban, urban and rural areas and with more than 10,000 customers each. Optimization is performed using a discrete adaptation of the Pareto Particle Swarm Optimization method proposed in [24] for real-valued variables. The validity of the proposed algorithm is verified by benchmarking it against an exhaustive search for one of the proposed load scenarios and networks. In summary, the key novel contributions of this paper are:

- The first comprehensive assessment of the potential tradeoff between MV losses and LV CVR energy savings when employing active voltage management of realistically modelled UK residential MV/LV networks (incorporating 5\%-7\% commercial/industrial loads).

- The comparison of four different infrastructure investment dependent operating scenarios, from low-flexibility, low investment (business as usual) networks, to highly flexible, high investment, OLTC equipped networks, with regard to their impact on CVR and MV losses and the trade-off between them.

- A discrete implementation of the real-valued Pareto Particle Swarm Optimization algorithm proposed in [24].

The remainder of the paper is organized as follows. Section II presents the load modelling and optimization methodology and the PPSO algorithm employed. Section III introduces the experimental setup, including the case study MV/LV networks. The experimental results are presented in Section IV. This is followed by a discussion of the results and conclusions in Section V. Throughout the paper various acronyms are used. For the reader's convenience the most frequently occurring acronyms are defined in Table I.

\section{Methodology}

\section{A. Base case load model}

CVR is a voltage management approach that relies on the voltage dependency of appliances to save energy. Hence, to simulate the impact of CVR voltage dependent load models must be incorporated into distribution network simulations. The standard practice is to employ a time-varying ZIP $(Z=$ constant impedance, $I=$ constant current and $P=$ constant power) load model [25]. In our simulations a resolution of one minute is used for the ZIP models of each individual appliance over a full day. Using the corresponding ZIP parameters of each appliance, the real power of the $m$-th appliance during the $t$-th time interval is given by

$$
P_{m}(t)=P_{o_{m}}(t)\left[\hat{Z}_{m}(t)\left(\frac{V(t)}{V_{o}}\right)^{2}+\hat{I}_{m}(t)\left(\frac{V(t)}{V_{o}}\right)+\hat{P}_{m}(t)\right]
$$

TABLE I. DEFINITION OF FREQUENTLY USED ACRONYMS

\begin{tabular}{|l|l|}
\hline CVR & Conservation Voltage Reduction \\
\hline ENWL & Electricity North West Ltd. (in the UK) \\
\hline LV & Low Voltage (Network) \\
\hline LVE & Low voltage network energy consumption \\
\hline MV & Medium Voltage (Network) \\
\hline MVL & Medium voltage network energy losses \\
\hline NLTC & No-load tap changer (transformer) \\
\hline OLTC & On-load tap changer (transformer) \\
\hline PPSO & Pareto Particle Swarm Optimization \\
\hline PSO & Particle Swarm Optimization \\
\hline ZIP & $\begin{array}{l}\text { Constant impedance (Z), constant current (I) } \\
\text { and constant power (P) (load model) }\end{array}$ \\
\hline
\end{tabular}

where, $P_{m}(t)$ and $P_{o_{m}}(t)$ denote the consumed active power for the current voltage $V(t)$ and the nominal voltage $V_{o}$, respectively, and parameters $\hat{Z}_{m}(t), \hat{I}_{m}(t)$ and $\hat{P}_{m}(t)$ represent constant impedance, constant current and constant power load dependencies, for each consumer at each sample instant.

ZIP models reflect the impact of voltage variation on the load from the perspective of the grid, and not the consumer. Therefore, matters regarding the most efficient operation of the devices being powered and customer experience are not factored in when employing CVR. For some devices, efficiency increases, and degradation decreases as the voltage drops. For others, the opposite applies. Consumers may experience slightly dimmer lights, or slightly longer times to boil their kettles, but it is generally considered that they are relatively insensitive to these variations [26].

\section{B. Aggregated ZIP load models}

To reduce the computational overhead associated with simulating the distribution networks considered in this study aggregated ZIP load models are introduced for the individual LV networks. The aggregated models are obtained by first aggregating the ZIP parameters of all appliances for each consumer to yield consumer level ZIP load models; then the ZIP models of all consumers connected to a given LV network are aggregated to yield the overall LV network ZIP load model. The aggregated ZIP parameters at each stage are computed using the following equations:

$$
\begin{gathered}
Z_{a g g}(t)=\frac{\sum_{m} \widehat{Z}_{m}(t) \cdot P_{o_{m}}(t)}{\sum_{m} P_{o_{m}}(t)} ; \\
I_{a g g}(t)=\frac{\sum_{m} \widehat{I}_{m}(t) \cdot P_{o_{m}}(t)}{\sum_{m} P_{o_{m}}(t)} ; \\
P_{a g g}(t)=\frac{\sum_{m} \widehat{P}_{m}(t) \cdot P_{o_{m}}(t)}{\sum_{m} P_{o_{m}}(t)}
\end{gathered}
$$

where, $Z_{a g g}, I_{a g g}$ and $P_{a g g}$ are the aggregated ZIP model parameters for active power of a given consumer (or LV network), $t$ denotes the $t$-th time interval and $m$ denotes the $m$-th appliance (or consumer). Equivalent expressions apply for reactive power load models [25].

\section{Optimization methodology}

To explore the trade-off that may exist between the two objectives - minimizing MV network power losses (MVL) and minimizing LV network energy consumption (LVE), a multi- 
objective optimization framework is required. This allows the Pareto front defining the trade-off between objectives to be determined. A typical multi-objective optimization problem has the form:

$$
\min _{\boldsymbol{x}} \boldsymbol{F}(\boldsymbol{x})=\left(f_{1}(\boldsymbol{x}), f_{2}(\boldsymbol{x}), \ldots, f_{n}(\boldsymbol{x})\right) \quad \text { s.t. } \boldsymbol{x} \in S,
$$

where $n$ is the number of scalar objective functions and $\boldsymbol{x}$ is the vector of decision variables, with a domain of definition $S$. For each $\boldsymbol{x} \in S$ there is a corresponding $\boldsymbol{F}(\boldsymbol{x}) \in C$, where $C$ is referred to as the objective space [27].

1) CVR optimization problem formulation

For the CVR optimization problem at hand, $n=2$, $f_{1}(\boldsymbol{x})=M V L(\boldsymbol{x})$ and $f_{2}(\boldsymbol{x})=\operatorname{LVE}(\boldsymbol{x})$ in (3), where $M V L(\boldsymbol{x})$ and $\operatorname{LVE}(\boldsymbol{x})$ are the MV network energy loss and LV network energy consumption objective functions, respectively, and $\boldsymbol{x}$ is the vector of network device parameters that can be manipulated by CVR in order to control the voltage levels on the network. For capacitors $x_{j} \in \boldsymbol{x}$ is an integer value from 0 to $C_{\max }$, while for transformers it is an integer corresponding to the tap position from 1 to $\operatorname{Tap}_{\max }$.

The MV network energy losses are computed as the sum of the energy losses at the MV transformers and medium voltage lines that is:

$$
\operatorname{MVL}(\boldsymbol{x})=\Delta P^{L}+\Delta P^{T r}
$$

where the $\triangle P \mathrm{~s}$ are the energy losses in the MV lines $(L)$ and transformers $(\mathrm{Tr})$. The LV network energy consumption is defined as:

$$
\operatorname{LVE}(\boldsymbol{x})=\sum_{m=1}^{N L}\left(P_{m}\right)+\Delta P^{L V}
$$

where $P_{m}$ is the active power consumption of the $m$-th consumer, $N L$ is the total number of consumers, and $\Delta P^{L V}$ represents the power losses in the LV network.

The optimization is subject to the constraint that the voltage along each feeder at each secondary bus should be within specified limits, that is:

$V_{i, \min }<V_{i}<V_{i, \max }$ at the $i^{\text {th }}$ monitoring point

This can be expressed mathematically as:

$$
\min _{\boldsymbol{x}} \boldsymbol{F}(\boldsymbol{x}) \text { s.t. } \quad\left\{\begin{array}{l}
g_{i}(\boldsymbol{x})<0 \\
h_{i}(\boldsymbol{x})<0
\end{array}, i=1 \text { to } N V\right.
$$

where $g_{i}(\boldsymbol{x})=V_{i}(\boldsymbol{x})-V_{i, \max }$ and $h_{i}(\boldsymbol{x})=V_{i, \min }-V_{i}(\boldsymbol{x})$. Here $g_{i}(\boldsymbol{x})$ and $h_{i}(\boldsymbol{x})$ define the constraints on the upper and lower limits of the permissible voltage levels, respectively. The network power flows and the associated power losses and voltage levels needed to compute $\boldsymbol{F}(\boldsymbol{x}), g_{i}(\boldsymbol{x})$ and $h_{i}(\boldsymbol{x})$ for a given $\boldsymbol{x}$ are obtained by simulating the three-phase network models for the control device settings specified by $\boldsymbol{x}$.

Various approaches exist to handle constraints in nonlinear optimization problems. Here, an unconstrained optimization problem is obtained by incorporating the constraints as additional penalty terms in each objective function, that is:

$$
f_{i}(\boldsymbol{x}) \rightarrow f_{i}(\boldsymbol{x})+P F(\boldsymbol{x})
$$

The penalty term, $P F(\boldsymbol{x})$, takes the form

$$
P F(\boldsymbol{x})=\sum_{i=1}^{N V}\left(\xi_{i}^{H}\left|g_{i}(\boldsymbol{x})\right|_{+}+\xi_{i}^{L}\left|h_{i}(\boldsymbol{x})\right|_{+}\right)
$$

where $\xi_{i}^{H}$ and $\xi_{i}^{L}$ are the penalty coefficients for higher and lower band voltage violations, respectively, for the $i$-th monitoring point. If a system is not experiencing any voltage violations, $P F(\boldsymbol{x})$ will be equal to zero. Penalty terms for other constraints such as loading limits on transformers and power lines can be included in a similar fashion if needed.

\section{2) Pareto Particle Swarm Optimization (PPSO)}

To solve the multi-objective discrete nonlinear CVR optimization problem we employ a modified implementation of the Pareto Particle Swarm Optimization (PPSO) technique proposed in [24]. This is one a number meta-heuristic optimization techniques frequently used to solve complex multi-objective optimization problems where conventional approaches are infeasible or do not scale well [28]. We selected it for our study due to its relative simplicity and good performance in many practical applications, including power systems applications such as sizing and placement of DG units for loss minimization, improving voltage profiles, etc. [29]. Particle Swarm Optimization (PSO) is a population-based optimization method inspired by the local and global interactions that occur in flocks of animals such as birds and fish [30] to achieve coordinated movement when searching for food. At each iteration of the swarm (flock), individuals, referred to as particles, update their velocities and positions based on the best experience of the swarm $\boldsymbol{p}_{g}(k)$ and their own best experience $\boldsymbol{p}_{i}(k)$. Conventional PSO is designed to solve single objective optimization problems. PPSO, its extension to multi-objective problems [24], is defined as follows for the case of two objectives $f_{1}(\boldsymbol{x})$ and $f_{2}(\boldsymbol{x})$ and a population size of $N_{p}$ particles:

1) Initialize the particle swarm by setting iteration number $k=1$ and randomly initializing the position and velocity of each particle in the swarm $\left(\boldsymbol{x}_{i}(k), \boldsymbol{v}_{i}(k), \quad i=\right.$ $\left.1, \ldots, N_{p}\right)$.

2) Evaluate fitness $f_{1}(\boldsymbol{x})$ and $f_{2}(\boldsymbol{x})$ for each particle.

3) Calculate the best individual solutions for each particle with respect to each objective function, $\boldsymbol{p}_{i 1}(k)$ and $\boldsymbol{p}_{i 2}(k)$, where $i$ is the particle index and $k$ is the iteration number.

4) Calculate the best global solutions for each objective function: $\boldsymbol{p}_{g 1}(k)$ and $\boldsymbol{p}_{g 2}(k)$.

5) Calculate the "average" of the two best global solutions $\boldsymbol{p}_{g}(k)$ and the distance between them $\boldsymbol{d} \boldsymbol{p}_{g}(k)$, that is:

$$
\begin{aligned}
& \boldsymbol{p}_{g}(k)=\left(\boldsymbol{p}_{g 1}(k)+\boldsymbol{p}_{g 2}(k)\right) / 2 \\
& \boldsymbol{d}_{g}(k)=\left\|\boldsymbol{p}_{g 1}(k)+\boldsymbol{p}_{g 2}(k)\right\|_{\mathbf{2}}
\end{aligned}
$$

Calculate the distance $\boldsymbol{d p}_{i}(k)$ between $\boldsymbol{p}_{i 1}(k)$ and $\boldsymbol{p}_{i 2}(k)$ for each particle, $i=1, \ldots, N_{p}$.

6) Calculate the best individual solution $\boldsymbol{p}_{i}(k)$ according to the following rules:

$$
\begin{aligned}
& \text { If } \boldsymbol{d p}_{i}(k)<\boldsymbol{d} \boldsymbol{p}_{g}(k) \\
& r_{i} \sim U(0,1) \\
& \text { else } \\
& \boldsymbol{p}_{i}(k)=r_{i} \boldsymbol{p}_{i 1}(k)+\left(1-r_{i}\right) \boldsymbol{p}_{i 2}(k)
\end{aligned}
$$




$$
\text { end } \boldsymbol{p}_{i}(k)=\frac{N-k}{N} \boldsymbol{p}_{i 1}(k)+\frac{k}{N} \boldsymbol{p}_{i 2}(k)
$$

Here, $r_{i}$ is a random number in the interval $[0,1], k$ is the $k$-th iteration and $N$ is the maximum number of iterations performed.

7) Using the computed values of $\boldsymbol{p}_{g}(k)$ and $\boldsymbol{p}_{i}(k)$ update the velocity of each particle.

8) Update the position of each particle $\boldsymbol{x}_{\boldsymbol{i}}(k)$ provided the updated position improves on one or both of $f_{1}\left(\boldsymbol{p}_{i 1}(k)\right)$ and $f_{2}\left(\boldsymbol{p}_{i 2}(k)\right)$. Otherwise retain the existing position.

9) If the termination condition (normally a pre-specified maximum number of iterations) is achieved then stop, otherwise set $k=k+1$ and go to step 2).

10) The Pareto set (front), $\boldsymbol{S}$, is then obtained as the union of all $\boldsymbol{p}_{i 1}(k)$ and $\boldsymbol{p}_{i 2}(k)$

The algorithm as written operates in the continuous domain. We adapt it to the discrete domain by rounding each variable to the nearest integer within the fitness function evaluations, that is: $f_{i}(\boldsymbol{x}) \rightarrow f_{i}(\operatorname{round}(\boldsymbol{x}))$. Likewise, $S \rightarrow \operatorname{round}(\boldsymbol{S})$.

\section{3) Pareto front and optimum solution}

The Pareto front captures the trade-off that exists between objectives, in this case MVL and LVE, with the final choice on the optimal solution dictated by the relative importance the end-user places on one objective over another. If the relative importance of $f_{1}(\boldsymbol{x})$ over $f_{2}(\boldsymbol{x})$ is denoted as $\beta$ then the multiobjective CVR optimization problem reduces to a single objective problem with the objective defined as:

$$
f_{\beta}(\boldsymbol{x})=\beta f_{1}(\boldsymbol{x})+f_{2}(\boldsymbol{x})
$$

As the units of both objective functions are the same $(\mathrm{kWh}), \beta$ is a dimensionless scalar between 0 and infinity. Since $f_{\beta}(\boldsymbol{x})=c$, where $c$ is a constant, generates linear contours in the objective function space (i.e. when plotting LVE versus MVL as in Fig. 1) with slope $-\beta$ and intercept $c$, it follows that the minimum value of $c$ occurs for the contour that is tangential to the Pareto front and that the corresponding tangent point represents the optimal settings.

\section{EXPERIMENTAL SETUP}

To explore the potential trade-off between the two performance measures of interest, three validated large $\mathrm{MV} / \mathrm{LV}$ networks (each with more than 10,000 customers) from the Smart Street networks were selected as case studies. The networks, which correspond, respectively, to dense urban, urban and rural areas, have been selected by ENWL to be representative of the range of circuit types in their distribution network in terms of customer density and type, physical and electrical constraints, LV interconnection, etc. [31]. The dense urban networks have all underground cables, the urban networks are predominantly underground cables but with some overhead lines, and the rural networks are predominantly overhead lines. All networks are operated as radial networks and serve primarily residential customers. The basic information for the selected networks is summarized in TABLE
II. As part of the trials one LV network in each area was equipped with an OLTC transformer with the remaining networks served by NLTC (no load tap changer) transformers.

The OLTC transformers installed on the primary side of the MV substations can adjust their voltage between $-17.16 \%$ and $+5.72 \%$ of the nominal level in 17 steps of $1.43 \%$, while the LV OLTC transformers (secondary transformers) can adjust the secondary voltage from $-8 \%$ to $+8 \%$ of the nominal setting in 9 steps of $2 \%$. In the UK, the nominal distribution transformer ratio (middle tap) is typically $11 \mathrm{kV}$ (or $6.6 \mathrm{kV}$ ) to $433 \mathrm{~V}$, which represents a natural boost of $8.25 \%$ with respect to the nominal voltage of $400 \mathrm{~V}$. The output voltage of the NLTC transformers can be manually adjusted from $-5 \%$ to $+5 \%$ of the nominal setting in 5 steps of $2.5 \%$.

TABLE II. CHARACTERISTICS OF THE CASE STUDY NETWORKS [31].

\begin{tabular}{|l|lll|}
\hline Network: & $\begin{array}{l}\text { Dense } \\
\text { Urban }\end{array}$ & Urban & Rural \\
\hline Voltage Level (kV): & $33 / 6.6$ & $33 / 11$ & $33 / 11$ \\
\hline $\begin{array}{l}\text { Primary Transformer } \\
\text { (MVA): }\end{array}$ & $2 \times 10$ & $3 \times 10$ & $2 \times 7.5$ \\
\hline Secondary Transformers (V) & $6600 / 433$ & \multicolumn{2}{|c|}{$11000 / 433$} \\
\hline Number of LV Networks: & 52 & 131 & 290 \\
\hline Number of Customers: & 10303 & 17068 & 10214 \\
\hline Residential (\%) & 94 & 95 & 93 \\
\hline Commercial/industrial (\%) & 6 & 5 & 7 \\
\hline MV OLTC & 2 & 3 & 2 \\
\hline LV OLTC & 1 & 1 & 1 \\
\hline LV NLTC & 51 & 130 & 289 \\
\hline MV Capacitors (kvar) & $1 \times 200$ & $1 \times 500$ & $2 \times 500$ \\
\hline
\end{tabular}

OpenDSS [32] was used to model and simulate the operation of the case study networks for representative winter and summer weekday load scenarios. The LV networks are modelled down to the level of individual households, with the household loads represented are single phase ZIP load models, while industry loads are connected as three phase loads. The simulations were performed at a resolution of 1 minute in terms of load profiles, which were generated using a modified version of the CREST tool as described in [33], while voltage control device optimization with respect to CVR was performed on a half hourly basis to be consistent with actual operation in the Smart Street trials [22]. Thus, the control device settings remain at fixed values for each half hour period, with the settings selected through optimization to be those which give, on average, the best performance over the corresponding 30 load profiles. The details for the network and load modelling can be found in [34]. Matlab was employed as a wrapper program to update the load values and voltage control device settings, execute the simulations at each iteration, and perform closed loop CVR using PPSO.

The voltage constraints adopted are as specified in the BS EN50610 standard. This states that $95 \%$ of the values of the 10 minutes moving average of the voltages at the LV level should fall within $+10 \%$ and $-6 \%$ of the nominal voltage level (230V line-to-neutral) and it should never drop below $-15 \%$. The voltage limits for the MV networks are specified as $\pm 6 \%$ of the nominal voltage $(6.6 \mathrm{kV}$ and $11 \mathrm{kV}$, line-to-line $)$.

To quantify the performance of CVR it is benchmarked against nominal operating conditions, i.e., where all the network control devices are fixed at their nominal settings. The nominal settings are defined as follows: (1) The taps of all 
MV OLTC transformers are allowed to change continuously to maintain 1 p.u. at the busbar; (2) The taps of all the OLTC and NLTC LV transformers are fixed at their middle location; (3) The MV capacitor banks are switched off.

The optimizations were performed using the PPSO algorithm with $N_{p}=50$ and $N=150$. These settings were determined following several trial runs. The number of iterations $N$ was selected as a value greater than the maximum number of iterations to convergence observed for the trial runs. There is always a risk that PPSO will be terminated prematurely when using a fixed number of iterations, but we have to balance this with the computational cost of running the algorithm for longer since the run-time grows linearly with the number of iterations. Based on prior experience, and the validation experiments presented in Section $\operatorname{IV}(B)$, we have some confidence that the chosen settings yield optimal or near-optimal solutions.

\section{Simulation Results}

\section{A. Scenarios}

To assess the full potential of voltage control devices with regard to their impact on MV energy loss and LV energy consumption, we investigate four different operating scenarios with regard to the availability and flexibility of voltage control infrastructure on the distribution network as follows:

Scenario 1 (actual network settings): The 3 LV OLTCs and the MV OLTCs are optimized every 30 minutes while the LV NLTCs are fixed at their middle tap. The MV capacitors operate in on/off mode (i.e. on at $100 \%$ of capacity or off) and are optimized on a half-hourly basis.

Scenario 2: The MV capacitors are disabled (switched off). The three LV OLTCs are replaced by NLTCs such that all the LV networks are supplied by NLTCs. Then the tap positions of the LV NLTCs are optimized on a daily basis for each sample day (winter and summer weekdays) while the tap position of the MV OLTCs are optimized every half-hour.

Scenario 3: All the LV NLTCs are replaced with OLTCs with the tap settings belonging to each one optimized on a half-hourly basis. The MV OLTCs are replaced by NLTCs and optimized on a daily basis (i.e. one setting applied for the full day). The MV capacitors are disabled (switched off).

Scenario 4 (most flexible): All the transformers on the LV and MV networks are operated as OLTCs and the MV capacitors are enabled and able to provide continuous car support over their full operating range. All devices are optimized on a half-hourly basis.

Scenario 1, which reflects the infrastructure available on Smart Street project case study networks, offers the least flexibility, while Scenario 4 offers the most flexibility with regard to controlling voltages on the network. Scenarios 2 and 3 fall between these extremes and have been included to assess the relative merits of voltage control flexibility at the MV versus LV level.

\section{B. Validation of the discrete PPSO implementation}

In this section we validate the performance of the proposed discrete PPSO algorithm by benchmarking its performance against an exhaustive search for the global optimum CVR settings for the summer weekday, dense urban network case study under Scenario 1 operating conditions.

Note that an exhaustive search was undertaken for only one scenario as evaluating all possible control action combinations to find the Pareto set is computationally very expensive and becomes prohibitive as the number of variables increases. The selected study had 5202 possible control device combinations and took over 3 days to complete on a standard PC (Intel $3.40 \mathrm{GHz}$ i7-3770 CPU, 8 GB Ram). It is estimated that an exhaustive search for the largest problem (Scenario 4) would take more than $10^{54}$ days to complete. In contrast PSO took 540 minutes to converge to the Pareto front in the Scenario 1 study and 840 minutes for the largest Scenario 4 problem. Thus, PSO is many orders of magnitude faster than an exhaustive search for the networks considered in our study, but in common with many other optimization methods it does not scale well as the dimensionality of the problem increases although it is recognized as among the most promising metaheuristic techniques in terms of scalability to high dimensional problems [35]. The scalability problem is compounded by the fact that in addition to the increase in the dimensionality of the optimization problem, larger networks require much greater simulation time per particle evaluation in PSO. However, both PSO and OpenDSS are amenable to parallel implementation [36], [37] which can be exploited to reduce computation time and enable application to much larger networks if needed.

Fig. 1 shows the relationship that exists between MVL and LVE for all feasible solutions to the CVR problem (as computed by an exhaustive evaluation of device control setting combinations) for three half-hour intervals from Scenario 1 (summer day). The selected intervals, which are representative of the patterns observed over the full day, are nighttime off-peak (4 am), midday (12 pm), and evening peak $(6 \mathrm{pm})$, as given in plots (a), (b) and (c), respectively. The final plot (d) in Fig. 1 is a zoomed-in plot of Fig. 1 (c) with the same scale used on both the MVL and LVE axes. The feasible solutions are shown as red dots in each plot. The PPSO estimated Pareto set is also shown in each plot using black square markers with magenta inset. These coincide exactly with the true Pareto set, as determined from the exhaustive search approach, hence confirming the correctness of the PPSO algorithm.

As introduced in Section II.3, the dashed green line in each plot in Fig. 1 is the minimum value contour, and the green dot is its tangent point with the Pareto front, for the relative weighting cost function, $f_{\beta}(\boldsymbol{x})$, when $\beta=1$. This represents the optimum solution when MVL and LVE are given equal weighting in the optimization problem and $f_{\beta}(\boldsymbol{x})$ is simply the Total Energy Consumed (TEC) by the distribution network.

Comparing results for each half-hour, it can be seen that the LVE - MVL trade-off varies with the time of day as a result of the associated variation in load on the network. In particular, at times the minimum MVL solution equates to the minimum TEC solution, at other times it equates to the minimum LVE solution, and at other times it differs from both these extremes. However, in practical terms, the much larger scale and variation in LVE compared to MVL means that the minimum TEC solution is dominated by minimization of the LVE component. 


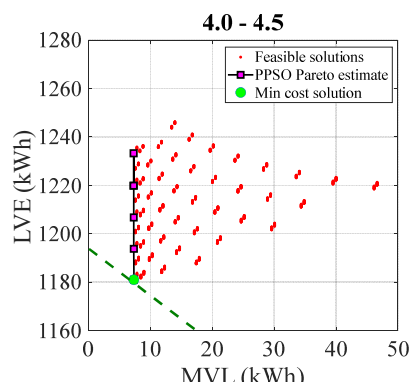

(a)

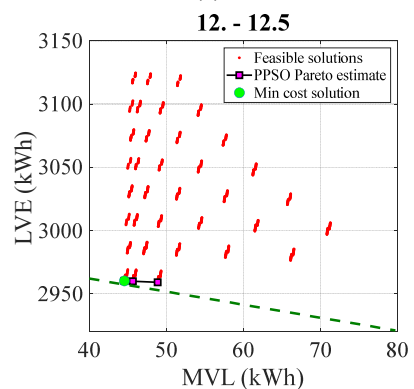

(b)

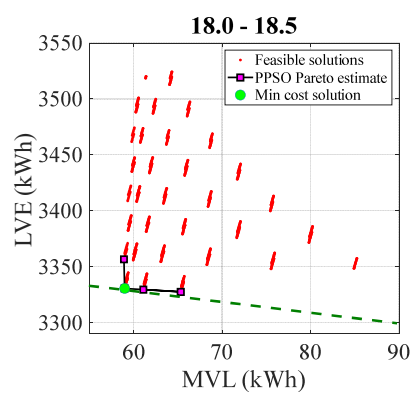

(c)

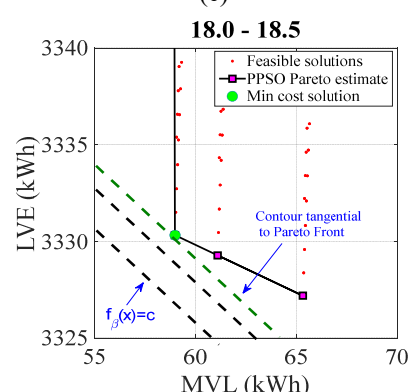

(d)
Fig. 1. A visualization of the trade-off between LVE and MVL at: (a) 4 am (off-peak); (b) 12 pm (midday), and (c) 6 pm (evening peak). Plot (d) is a zoomed-in version of plot (c) with the same scale used for both the LVE and MVL axes, and with the contours for equally weighted objectives illustrated.

Fig. 2 is a zoomed in graph of the 5 points that define the Pareto front in Fig. 1 (a). The $\beta$ value recorded on the graph for each point is the slope of the line between that point and the point to its right on the Pareto Front. These values give an indication of the sensitivity of the solution to the relative weighting between objectives as they represent the weighing that must be applied in order for the contact point between $f_{\beta}(\boldsymbol{x})=c$ and the Pareto front to change from one point to the next, representing a change in the optimal control settings, $\boldsymbol{x}$. Thus, for this example, the optimum control settings remain the same for $0 \leq \beta<667$. This range includes both the minimum LVE and minimum TEC objectives. The second point is the solution for $667 \leq \beta<1083$, and so on for the other points until we reach the point furthest to the left. This point is the minimum MVL solution $(\beta=\infty)$ and remains unchanged for $1400<\beta \leq \infty$. The $\beta=1$ range yielding the same control settings as the minimum TEC solution for the midday and evening peak optimization problems are respectively, $0.4 \leq \beta<\infty$ and $0.3 \leq \beta<1552$.

The invariance in the optimal settings over such large ranges of $\beta$ indicates that solutions are relatively insensitive to changes in the relative importance of $f_{1}(\mathbf{x})$ over $f_{2}(\mathbf{x})$. Thus, while we may wish to consider values of $\beta>1$ to reflect indirect costs to the operator of MVL, such as asset maintenance costs linked to power loss related degradation, these are unlikely to be of a scale to substantially alter the optimal solution because of the dominance of LVE over MVL.

As a final observation we note that if minimizing LVE is prioritized $(\beta=0)$, the increase in MVL is of the order of only $0.04 \mathrm{kWh}$ for a reduction in LVE of $50 \mathrm{kWh}$ when compared to the minimum MVL solution $(\beta=\infty)$.

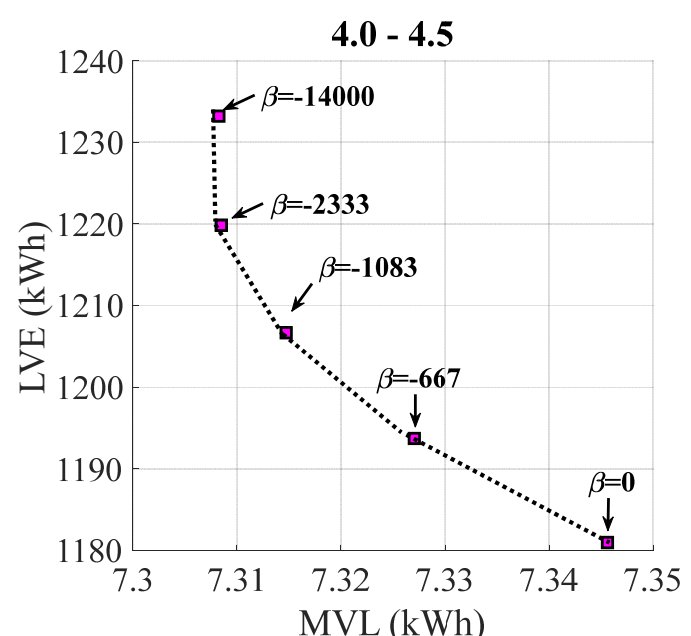

Fig. 2. The trade-off between MVL and LVE with CVR at 4 a.m. on the dense urban network operating under Scenario 1 (summer weekday).

To verify the performance of PPSO for larger problems where an exhaustive search is not possible, a comparative study was also undertaken between PPSO and a multiobjective genetic algorithm (MOGA) optimizer for the rural network operating under Scenario 2. MOGA is an alternative well-established multi-objective method which is available as part of MATLAB's global optimization toolbox [38]. This was implemented using the default toolbox settings, and with $N_{p}=$ 50 and $N=150$, and found to yield similar results to PPSO (i.e. similar Pareto sets) providing further assurance of the efficacy of the discretized PPSO algorithm. By way of example, Fig. 3 shows the Pareto sets obtained using both approaches for the off-peak (4 am) and peak $(6 \mathrm{pm})$ half hours. The advantage that PPSO has over MOGA is that it is usually faster to compute. [39]. Here PPSO was $10 \%$ a faster than MOGA taking 108 mins to compute both Pareto fronts in Fig. 3 on an Intel(R) Core(TM) i5-2520M CPU @ 2.50GHz $2.5 \mathrm{GHz}$ laptop.

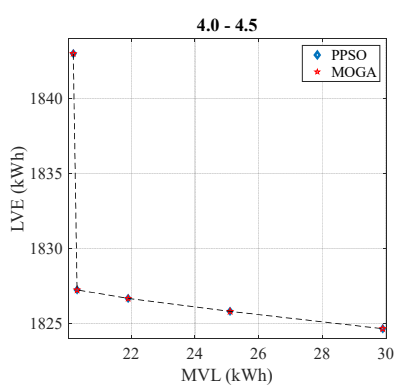

(a)

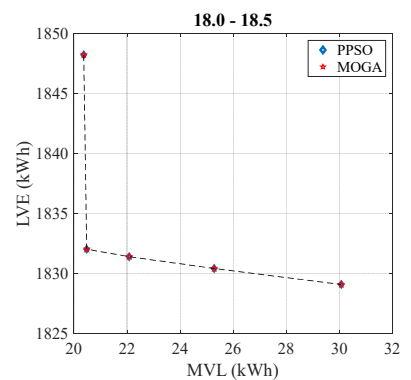

(b)
Fig. 3. LVE and MVL Pareto Sets identified by PPSO and MOGA for 4 a.m. (off-peak) and $6 \mathrm{pm}$ (evening peak) loads for the MV/LV rural network operating under Scenario 2 conditions.

\section{Illustration of the optimum solution (Scenario 1)}

As an illustration of the validity of the optimized CVR device settings obtained with PPSO, in this section we present the control settings and resulting minimum voltage levels obtained for each half hour over the full summer and winter weekday load conditions for the dense urban network operating under Scenario 1. In each case the selected Pareto optimized settings are the minimum TEC solution $(\beta=1)$. 
The 10-minute moving average of the minimum voltage before and after optimization are plotted in Fig. 4 (a) and (c) for summer and winter weekdays, respectively. The corresponding tap positions for the OLTC and NLTC transformers and the switching patterns of the MV capacitor are presented in Fig. 4 (b) and (d) for the summer and winter weekdays, respectively. It can be observed that the voltage level is regulated close to its lower limit of 0.94 p.u. over both days with the MV capacitor helping to recover the voltage drop when needed. This is much more often during the winter weekday scenario due the substantially greater loading of the network during the winter.

\section{PPSO CVR performance and Trade-off Analysis}

In this section, we investigate the extent to which the patterns observed in Section IV-B apply for the trade-off between aggregate MVL and LVE over a full day for a range of network types, load profiles and operating conditions.

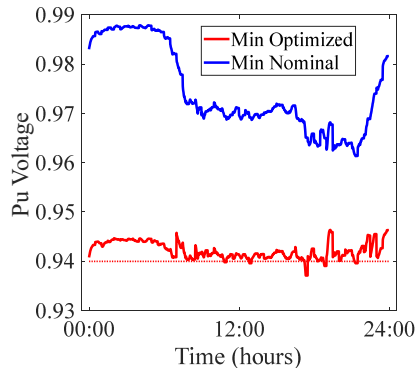

(a)

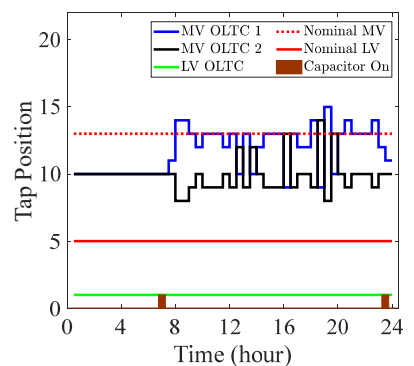

(b)

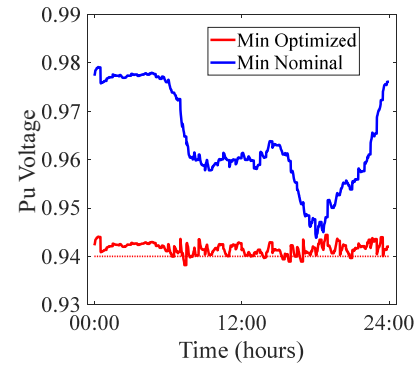

(c)

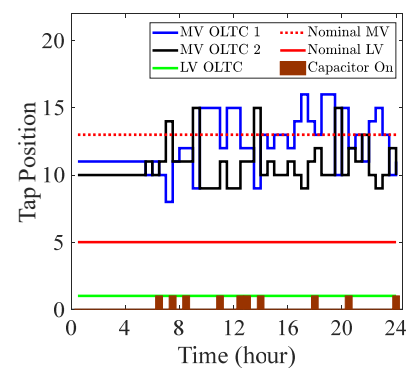

(d)
Fig. 4. (a) 10 minutes moving average of the minimum voltage on the dense urban network operating under Scenario I before and after CVR optimization with PPSO and (b) Control device settings (M/LV O/NLTC taps, MV and capacitor switching) for a summer weekday; (c) and (d) a winter weekday.

The trade-offs that exist are captured by tabulating the results for the min LVE, min MVL and min TEC optimization objectives. These correspond to the extremes of the Pareto front and the equal weighting solution, respectively.

TABLE III presents the results for summer and winter weekday load profiles for PPSO optimized CVR applied to the three case study networks defined in TABLE II operating under the four operating scenarios given in Section IV-A. Specifically, TABLE III shows the actual $(\mathrm{kWh})$ and percentage reduction in LVE, MVL and TEC relative to the values obtained under nominal operating conditions.

Considering the percentage reduction achieved with the different scenarios, it is apparent that the scenarios are in order of performance with scenario 1 yielding the smallest reductions in LVE, MVL and TEC and scenario 4 yielding the largest. This is simply a reflection of the fact that the operating scenarios are in order of increasing control flexibility, and hence increasing ability to manipulate network voltage profiles.

In practical terms, there is little to choose between scenarios 2,3 and 4 , but, since 3 and 4 require substantial infrastructure investment, scenario 2 may represent the most attractive proposition for distribution companies. Of course, scenario 2 comes with an increased operating overhead since the tap position of each LV NTLC has to be manually set to its optimal daily position for the different day types.

The very poor performance of scenario 1 relative to the other scenarios is a consequence of the tap settings for the LV NLTC transformers remaining fixed at their nominal (middle tap) position. This limits the ability of MV OLTCs to regulate the LV voltage levels, as they have to find the best compromise across all network types and loading levels. Furthermore, they are forced to operate at lower voltage levels to deliver CVR on the LV side, leading to increased losses on the MV networks. These losses largely cancel out the gains from the reduced power flow arising from CVR, hence the very low MVL reductions observed.

It can be concluded that optimizing the LV transformer tap positions, either once for each day type (scenario 2) or continuously (scenarios 3 and 4), is the largest contributor to the superior performance of the other scenarios. The remaining control settings, including the MV OLTCs and the MV capacitors, provide only minor improvements in comparison.

A comparison of the performance of PPSO optimized CVR on the three case study networks, as given in Fig. 5, reveals that on balance CVR performs similarly for both the rural and dense urban networks, with slightly greater reductions in LVE and MVL observed with the rural network in the winter and with the dense urban network in the summer, for all four operating scenarios. In contrast the reductions obtained on the urban network are between $20 \%$ and $30 \%$ less than those achieved on the other networks. This may be attributed to the fact that the urban network is much more heavily loaded than the other case study networks and, therefore, has less head room for CVR. It consumes 1.6 times more energy and generates MV losses than are 13 times greater than the other networks.

$\square$ Scenario $1 \quad$ Scenario $2 \square$ Scenario $3 \quad \square$ Scenario 4

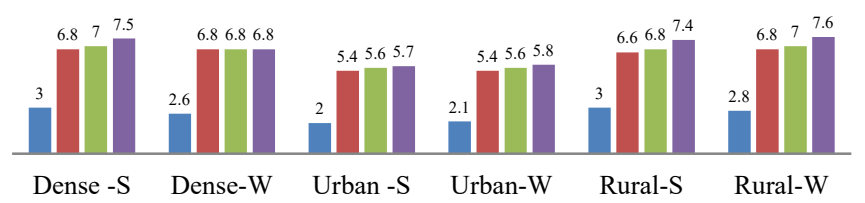

(a) \% Reduction in LVE

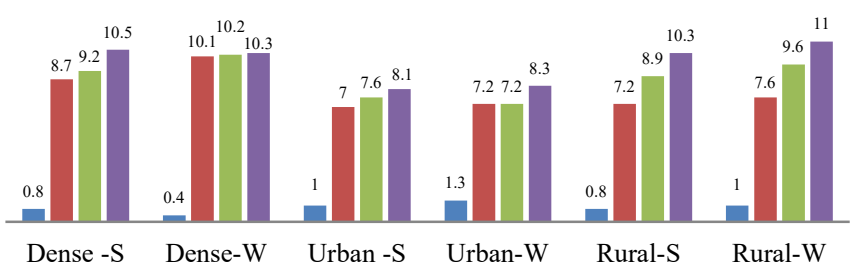

(b) \% Reduction in MVL

Fig. 5. Percentage reduction in LVE and MVL for each network for the different load profiles and operating scenarios considered for the min TEC solution ( $\mathrm{S}=$ summer, $\mathrm{W}=$ winter load profile) 
TABLE III. ACTUAL AND PERCENTAGE REDUCTION IN LVE, MVL AND TEC COMPARED TO THE NOMINAL SETTINGS ON SUMMER AND WINTER WEEKDAYS FOR THE CASE STUDY NETWORKS

\begin{tabular}{|c|c|c|c|c|c|c|c|c|c|c|c|c|c|c|}
\hline \multirow{3}{*}{ 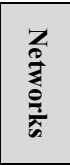 } & \multirow{3}{*}{ 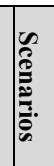 } & \multirow{3}{*}{ 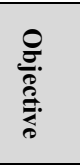 } & \multicolumn{6}{|c|}{ Summer } & \multicolumn{6}{|c|}{ Winter } \\
\hline & & & \multicolumn{2}{|c|}{$\begin{array}{l}\text { Min } \\
\text { LVE }\end{array}$} & \multicolumn{2}{|c|}{$\begin{array}{c}\text { Min } \\
\text { MVL }\end{array}$} & \multicolumn{2}{|c|}{$\begin{array}{c}\text { Min } \\
\text { TEC } \\
\end{array}$} & \multicolumn{2}{|c|}{$\begin{array}{c}\text { Min } \\
\text { LVE } \\
\end{array}$} & \multicolumn{2}{|c|}{$\begin{array}{c}\text { Min } \\
\text { MVL } \\
\end{array}$} & \multicolumn{2}{|c|}{$\begin{array}{c}\text { Min } \\
\text { TEC }\end{array}$} \\
\hline & & & $\begin{array}{c}\text { Real } \\
(\mathrm{kWh})\end{array}$ & $\%$ & $\begin{array}{c}\text { Real } \\
(\mathrm{kWh})\end{array}$ & $\%$ & $\begin{array}{c}\text { Real } \\
(\mathrm{kWh})\end{array}$ & $\%$ & $\begin{array}{c}\text { Real } \\
(\mathrm{kWh})\end{array}$ & $\%$ & $\begin{array}{c}\text { Real } \\
(\mathbf{k W h})\end{array}$ & $\%$ & $\begin{array}{c}\text { Real } \\
(\mathrm{kWh})\end{array}$ & $\%$ \\
\hline \multirow{12}{*}{ 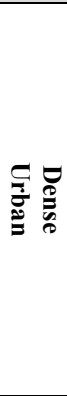 } & \multirow{3}{*}{1} & LVE & 3684 & 3.0 & 2796 & 2.2 & 3675 & 3.0 & 4238 & 2.6 & 3540 & 2.2 & 4238 & 2.6 \\
\hline & & MVL & 2 & 0.1 & 18 & 1.0 & 15 & 0.8 & 12 & 0.4 & 37 & 1.2 & 12 & 0.4 \\
\hline & & TEC & 3686 & 3.0 & 2814 & 2.2 & 3690 & 3.0 & 4250 & 2.6 & 3577 & 2.2 & 4250 & 2.6 \\
\hline & \multirow{3}{*}{2} & LVE & 8414 & 6.8 & 7939 & 6.4 & 8401 & 6.8 & 11080 & 6.8 & 10444 & 6.5 & 11032 & 6.8 \\
\hline & & MVL & 109 & 6.0 & 161 & 8.9 & 157 & 8.7 & 230 & 7.4 & 329 & 10.6 & 313 & 10.1 \\
\hline & & TEC & 8523 & 6.9 & 8100 & 6.5 & 8558 & 6.9 & 11310 & 6.9 & 10773 & 6.7 & 11345 & 7.0 \\
\hline & \multirow{3}{*}{3} & LVE & 8685 & 7.0 & 8352 & 6.7 & 8683 & 7.0 & 11082 & 6.8 & 10605 & 6.6 & 11077 & 6.8 \\
\hline & & MVL & 155 & 8.6 & 172 & 9.5 & 166 & 9.2 & 309 & 10.1 & 338 & 10.9 & 316 & 10.2 \\
\hline & & TEC & 8840 & 7.1 & 8524 & 6.8 & 8849 & 7.1 & 11391 & 7.0 & 10943 & 6.8 & 11393 & 7.0 \\
\hline & \multirow{3}{*}{4} & LVE & 9316 & 7.5 & 9117 & 7.3 & 9315 & 7.5 & 11085 & 6.8 & 10782 & 6.7 & 11082 & 6.8 \\
\hline & & MVL & 187 & 10.4 & 197 & 10.9 & 189 & 10.5 & 312 & 10.1 & 334 & 10.8 & 318 & 10.3 \\
\hline & & TEC & 9503 & 7.7 & 9314 & 7.5 & 9504 & 7.7 & 11397 & 7.0 & 11116 & 6.9 & 11400 & 7.0 \\
\hline \multirow{12}{*}{ 实 } & \multirow{3}{*}{1} & LVE & 3945 & 2.0 & 3353 & 1.7 & 3944 & 2.0 & 5476 & 2.1 & 4694 & 1.8 & 5455 & 2.1 \\
\hline & & MVL & 14 & 0.5 & 55 & 1.9 & 29 & 1.0 & 28 & 0.6 & 99 & 2.1 & 61 & 1.3 \\
\hline & & TEC & 3959 & 2.0 & 3408 & 1.7 & 3973 & 2.0 & 5504 & 2.1 & 4793 & 1.8 & 5516 & 2.1 \\
\hline & \multirow{3}{*}{2} & LVE & 10650 & 5.4 & 10058 & 5.1 & 10647 & 5.4 & 14079 & 5.4 & 13558 & 5.2 & 14068 & 5.4 \\
\hline & & MVL & 176 & 6.0 & 226 & 7.7 & 205 & 7.0 & 290 & 6.2 & 369 & 7.9 & 336 & 7.2 \\
\hline & & TEC & 10826 & 5.5 & 10284 & 5.2 & 10852 & 5.5 & 14369 & 5.5 & 13927 & 5.3 & 14404 & 5.5 \\
\hline & \multirow{3}{*}{3} & LVE & 11044 & 5.6 & 10400 & 5.2 & 11038 & 5.6 & 14622 & 5.6 & 14361 & 5.5 & 14619 & 5.6 \\
\hline & & MVL & 188 & 6.4 & 238 & 8.1 & 223 & 7.6 & 333 & 7.1 & 394 & 8.4 & 338 & 7.2 \\
\hline & & TEC & 11232 & 5.7 & 10638 & 5.3 & 11261 & 5.7 & 14955 & 5.7 & 14755 & 5.7 & 14957 & 5.7 \\
\hline & \multirow{3}{*}{4} & LVE & 11242 & 5.7 & 10453 & 5.3 & 11239 & 5.7 & 15122 & 5.8 & 14601 & 5.6 & 15118 & 5.8 \\
\hline & & MVL & 209 & 7.1 & 253 & 8.6 & 238 & 8.1 & 343 & 7.3 & 418 & 8.9 & 390 & 8.3 \\
\hline & & TEC & 11451 & 5.8 & 10706 & 5.4 & 11477 & 5.8 & 15465 & 5.9 & 15019 & 5.8 & 15508 & 5.9 \\
\hline & & LVE & 3577 & 3.0 & 2516 & 2.1 & 3573 & 3.0 & 4458 & 2.8 & 3632 & 2.3 & 4453 & 2.8 \\
\hline & 1 & MVL & 2 & 0.1 & 26 & 1.4 & 15 & 0.8 & 6 & 0.2 & 54 & 1.7 & 32 & 1.0 \\
\hline & & TEC & 3579 & 3.0 & 2542 & 2.1 & 3588 & 3.0 & 4464 & 2.8 & 3686 & 2.3 & 4485 & 2.8 \\
\hline & & LVE & 7844 & 6.6 & 7724 & 6.5 & 7815 & 6.6 & 10815 & 6.8 & 10338 & 6.5 & 10805 & 6.8 \\
\hline & 2 & MVL & 72 & 3.9 & 138 & 7.5 & 133 & 7.2 & 129 & 4.1 & 246 & 7.8 & 240 & 7.6 \\
\hline$\underset{\Xi}{\Xi}$ & & TEC & 7916 & 6.7 & 7862 & 6.6 & 7948 & 6.7 & 10944 & 6.9 & 10584 & 6.7 & 11045 & 7.0 \\
\hline 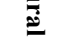 & & LVE & 8091 & 6.8 & 7843 & 6.6 & 8090 & 6.8 & 11133 & 7.0 & 10656 & 6.7 & 11129 & 7.0 \\
\hline & 3 & MVL & 162 & 8.8 & 169 & 9.2 & 164 & 8.9 & 286 & 9.1 & 306 & 9.7 & 303 & 9.6 \\
\hline & & TEC & 8253 & 6.9 & 8012 & 6.7 & 8254 & 6.9 & 11419 & 7.2 & 10962 & 6.9 & 11432 & 7.2 \\
\hline & & LVE & 8795 & 7.4 & 8675 & 7.3 & 8792 & 7.4 & 12087 & 7.6 & 11610 & 7.3 & 12083 & 7.6 \\
\hline & 4 & MVL & 186 & 10.1 & 199 & 10.8 & 190 & 10.3 & 330 & 10.5 & 353 & 11.2 & 347 & 11.0 \\
\hline & & TEC & 8981 & 7.6 & 8874 & 7.5 & 8982 & 7.6 & 12417 & 7.8 & 11963 & 7.5 & 12430 & 7.8 \\
\hline & & LVE & 7941 & 5.6 & 7436 & 5.2 & 7943 & 5.6 & 10440 & 5.5 & 9902 & 5.2 & 10430 & 5.5 \\
\hline Ave & & MVL & 122 & 5.7 & 154 & 7.1 & 144 & 6.7 & 217 & 6.1 & 273 & 7.6 & 251 & 7.0 \\
\hline & & TEC & 8062 & 5.7 & 7590 & 5.3 & 8078 & 5.7 & 10657 & 5.6 & 10175 & 5.4 & 10680 & 5.6 \\
\hline
\end{tabular}

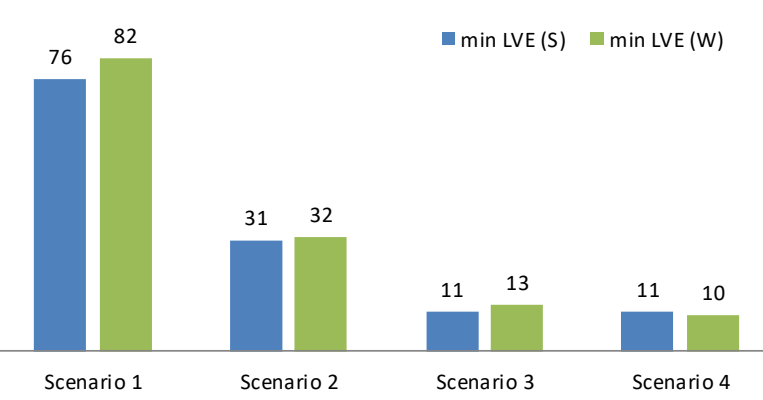

Fig. 6. Percentage trade-off in MVL with respect to its optimal value when selecting the min LVE solution ( $\mathrm{S}=$ summer, $\mathrm{W}=$ winter)

The bar chart in Fig. 6 shows the trade-off in MVL with respect to its optimal value when selecting the min LVE (i.e. optimum CVR) solution. The trade-off varies between $10 \%$ and $82 \%$ depending on the scenario and season. A striking trend in the chart is the reduction in trade-off with increasing scenario number. This correlates with the increasing ability of the networks to independently regulate the $\mathrm{MV}$ and $\mathrm{LV}$ voltage levels while performing CVR, thereby allowing higher voltage levels to be maintained on the MV networks.

It should be noted that, while the trade-off in MVL is significant, the corresponding reduction in LV energy consumption is much greater. The CVR energy saving averaged over all networks and day-types varies between 6.1 $\mathrm{kWh}$ and $21.5 \mathrm{kWh}$ per $1 \mathrm{kWh}$ increase in MV losses.

\section{DISCUSSION AND CONCLUSIONS}

Using three validated large MV/LV networks (each with more than 10,000 customers) from the Smart Street trial area and one-minute resolution time varying consumer load profiles representative of summer and winter weekdays in the UK, which were developed as part of ENWL's Ofgem funded 'Smart Street' project [22], a comprehensive investigation of multi-objective CVR optimization has been undertaken to explore the trade-off that exists between LV energy consumption (LVE) and MV energy losses (MVL). 
Four different CVR operating scenarios were considered representing difference levels of network flexibility with regard to the availability of assets that can be used for active voltage management (such as MV and LV OLTCs and MV capacitors). A discrete implementation of Pareto Particle Swarm Optimization (PPSO) was introduced as a scalable optimization methodology for CVR and validated against a full enumeration of all feasible solutions and an alternative genetic algorithm based multi-objective optimization method (MOGA) for selected networks and operating scenarios.

Our studies show that PPSO is an effective tool for finding the Pareto Front in the multi-objective CVR problem. More importantly, we conclude that a trade-off does exist between LVE and MVL reduction, the exact nature of which depends on the time of day and hence the loading of the network. This is a key finding as it allows distribution companies to determine the most appropriate investments and control strategies to maximize the benefit of CVR considering energy consumption and losses.

The results show that the optimal CVR solution is insensitive to the relative weighting network operators may place on the MVL and LVE objectives, with large changes in weightings required for there to be a material impact on the optimal voltage control device settings. This means that a precise estimate of the relative importance of MVL versus LVE is not required. Network operators can achieve optimal CVR performance with a negligible MVL downside by simply assuming an equal weighting between the objectives.

The LVE-MVL trade-off falls off with increasing network flexibility, with the availability of controllable LV transformers being the decisive factor in delivering effective CVR and reduced trade-off. In particular, if CVR is delivered through MV OLTC adjustment only with no facility to optimize LV transformer settings, the increased losses on the MV networks as a result of operating at lower voltages largely cancel out the gains from the reduced power flow arising from lower LV energy consumption, hence the very low MVL reductions observed for Scenario 1. In contrast, installing LV OLTC transformers on all LV networks (Scenario 4), makes it possible to simultaneously minimize MV losses and LV energy consumption, allowing the full potential for energy reduction to be realized. For the case study networks the difference in performance between Scenario 1 and 4 was a factor of 2.6 for LVE reduction and 9.6 for MVL reduction.

Overall, substantial energy savings can be achieved through active voltage management, but distribution companies will need to invest in LV OLTC transformers to realize its full potential, both in terms of maximizing LVE reductions and minimizing MVL trade-off.

We did not consider meshing in our study as the simulated networks, which were accurate representations of the actual Smart Street networks, are operated radially. In general, meshing would be expected to create greater capacity for CVR energy savings by virtue of having flatter volage profiles along interconnected LV feeders. However, this should not impact the trade-off with MV losses, which is largely dictated by the flexibility of the network in controlling the voltage levels. Delivering lower voltage levels on the LV side may require voltage levels to be reduced on the MV side which increases MV losses, irrespective of the network topology. Whether this is significant or not is then dictated by the scale of reduction in losses due to the overall reduction in power drawn by the LV networks relative to the increase in losses due to the drop in MV voltage levels. This is the case even if the network loads were largely ZI rather than ZIP (i.e. no constant power components and therefore no increase in current draw and associated line losses on the LV side).

The MV/LV voltage management methodology employed to achieve joint CVR and MV loss reduction makes no assumptions about the topology of the network, or the number or location of control devices and is therefore applicable to network topologies beyond the traditional radial networks of the case studies considered here, for example, to meshed networks where LV networks may be connected to more than one substation. The methodology as presented is intended for an off-line analysis of the trade-off that exist when employing active voltage management across MV/LV networks, providing system operators with guidance on the benefits of different forms of implementation/infrastructure investments. Determining the full Pareto front is computationally intensive and not amenable to real-time implementation. As such this is not an approach that could be used in real-time in its current form. It should also be noted that the CVR simulation studies assume idealized load profile predictions, and thus the results presented represent the best-case scenario for CVR. Assessing the impacts of errors in load predictions on the performance of CVR optimization either as a single objective or in conjunction with MV loss optimization, and developing strategies to mitigate these impacts, are important topics for future research.

OLTC transformers and capacitor banks were the only voltage control devices exploited for active voltage management in this study, reflecting the existing and proposed provision of such devices on the selected case study networks. The development of distributed flexible AC transmission system (D-FACTS) devices [40], [41], [42], and the proliferation of new low carbon technologies (LCTs) such as electric vehicles, distributed generation and prosumers with dispatchable loads, will offer distribution network operators other possibilities for active voltage management in the future [43], [44]. Assessing the performance and commercial viability of these new technologies in conjunction with, or as an alternative to, OLTC transformers and capacitor banks for joint CVR and MV loss optimization are also interesting topics for further research.

\section{ACKNOWLEDGMENT}

The authors gratefully acknowledge Electricity North West Limited (ENWL), UK, for providing access to their data.

\section{REFERENCES}

[1] W. Ellens, A. Berry, and S. West, "A quantification of the energy savings by Conservation Voltage Reduction", in 2012 IEEE International Conference on Power System Technology (POWERCON), Auckland, 2012.

[2] E. Diskin, T. Fallon, G. O'Mahony, and C. Power, "Conservation voltage reduction and voltage optimization on Irish distribution networks", in Integration of Renewables into the Distribution Grid, CIRED 2012 Workshop, Lisbon, 2012. 
[3] R. W. Uluski, Volt/VAR Control and Optimization Concepts and Issues. 2011. [Online]. Available: ias-research.com/en/typography/ item/643-volt-var-control-and-optimization-concepts-and-issues.

[4] R. Preiss and V. Warnock, "Impact of Voltage Reduction on Energy and Demand", IEEE Transactions on Power Apparatus and Systems, vol. 97, no. 5, pp. 1665-1671, 1978.

[5] J. Erickson and S. Gilligan, "The Effects of Voltage Reduction on Distribution Circuit Loads", IEEE Power Engineering Review, vol. 2, no. 7, pp. 39-40, 1982

[6] D. Lauria, "Conservation Voltage Reduction (CVR) at Northeas Utilities", IEEE Trans. Power Delivery, vol. 2(4), pp. 1186-1191, 1987.

[7] J. D. Steese, Assessment of Conservation Voltage Reduction Applicable in the BPA Service Region. United States: Bonneville Power Administration, 1987.

[8] S. Lefebvre, G. Gaba, A.O. Ba, D. Asber, A. Ricard, C. Perreault, and D. Chartrand, "Measuring the efficiency of voltage reduction at HydroQuebec distribution", in IEEE Power and Energy Society General Meeting, Pittsburgh, PA, USA, 2008.

[9] A. Dwyer, R. Nielsen, J. Stangl and N. Markushevich, "Load to voltage dependency tests at B.C. Hydro", IEEE Transactions on Power Systems, vol. 10, no. 2, pp. 709-715, 1995

[10] M. Manbachi, A. Sadu, H. Farhangi, A. Monti, A. Palizban, F. Ponci, and S. Arzanpour, "Real-Time Co-Simulation Platform for Smart Grid Volt-VAR Optimization Using IEC 61850”, IEEE Transactions on Industrial Informatics, vol. 12. no. 4, 1392-1402, 2016.

[11] R. Neal, "The use of AMI meters and solar PV inverters in an advanced Volt/VAr control system on a distribution circuit", in Proc. 2010 IEEE PES Transmission and Distribution Conf. Expo., 2010, pp. 1-4.

[12] T.Wilson and D. Bell, "Energy conservation and demand control using distribution automation technologies", in Proc. Rural Electric Power Conf., 2004, 2004, p. C4-1-12.

[13] M. A. Peskin, P. W. Powell, and E. J. Hall, "Conservation Voltage Reduction with feedback from Advanced Metering Infrastructure", in Transmission and Distribution Conference and Exposition (T\&D), Orlando, FL, 2012

[14] J. Wang, A. Raza, T. Hong, A. C. Sullberg, F. D. León and Q. Huang, "Analysis of Energy Savings of CVR Including Refrigeration Loads in Distribution Systems", IEEE Transactions on Power Delivery, vol. 33, no. 1, p.p. 158-168, Feb. 2018.

[15] V. Farahani, S. H. H. Sadeghi, H. A. Abyaneh, S. M. M. Agah and K Mazlumi, "Energy Loss Reduction by Conductor Replacement and Capacitor Placement in Distribution Systems", IEEE Transactions on Power Systems, vol. 28, no. 3, p.p. 2077-2085, August 2013.

[16] X. Su, M. A. S. Masoum, and P. J. Wolfs, "PSO and Improved BSFS Based Sequential Comprehensive Placement and Real-Time MultiObjective Control of Delta-Connected Switched Capacitors in Unbalanced Radial MV Distribution Networks", IEEE Transactions on Power Systems, vol. 31, no. 1, p.p. 612-622. January 2016.

[17] S. Satsangi, and G. B. Kumbhar, "Effect of load models on scheduling of VVC devices in a distribution network", IET Generation, Transmission \& Distribution., vol. 12. no. 17, 3993-4001, 2018.

[18] X. Zhang, A. J. Flueck, and C. P. Nguyen, "Agent-Based Distributed Volt/Var Control With Distributed Power Flow Solver in Smart Grid", IEEE Transaction on Smart Grid., vol. 7, no. 2, p.p. 600-607, 2016.

[19] P. Bagheri, and W. Xu, "Assessing Benefits of Volt-Var Control Schemes Using AMI Data Analytics", IEEE Transaction on Smart Grid., vol. 8, no. 3, p.p. 1295-1304, 2017.

[20] L. Gutierrez-Lagos, L. F. Ochoa, "OPF-based CVR Operation in PVRich MV-LV Distribution Networks", IEEE Transactions on Power Systems, vol. 34, no. 4, p.p. $2778-2789,23^{\text {rd }}$ Jan 2019

[21] M. R. Andervazh, J. Olamaei, and M. R. Haghifam, “Adaptive multiobjective distribution network reconfiguration using multi-objective discrete particles swarm optimization algorithm and graph theory", IET Gener. Transm. Distrib., vol. 7, no. 12, pp. 1367-1382, 2013.

[22] G. Paterson, "Final HV and LV Voltage and Configuration Optimization Study", Electricity North West Limited, Manchester: UK, 10 April 2018.

[23] H. Gharavi, X. Liu, and S. McLoone, "Multi-Objective Voltage Optimization Trade-off Assessment for Coupled HV and LV Networks", in 52nd International Universities' power Engineering Conference, Heraklion, Crete, Greece, 28th -31st August 2017.
[24] L.B. Zhang, C.G Zhou, X.H. Liu, Z.Q. Ma, M. Ma and Y.C. Liang, "Solving Multi Objective Optimization Problems Using Particle Swarm Optimization", in The Congress on Evolutionary Computation, Canberra, ACT, Australia, 8-12 Dec. 2003.

[25] W. W. Price, C. W. Taylor, and G. J. Rogers, "Standard load models for power flow and dynamic performance simulation", IEEE Transactions on Power Systems, vol. 10, no. 3, p.p. 1302-1313, 1995.

[26] H.L. Willis, (ed.), Distributed power generation: planning and evaluation. CRC Press, 2018

[27] K. Miettinen, Nonlinear Multiobjective Optimization, vol. 12, Springer, 1999.

[28] A. Gogna and A. Tayal, "Metaheuristics: review and application", Journal of Experimental \& Theoretical Artificial Intelligence, vol. 25, no .4, pp. 503-526, 2013.

[29] A. Ameli, S. Bahrami, F. Khazaeli and M.R. Haghifam, "A multiobjective particle swarm optimization for sizing and placement of DGs from DG owner's and distribution company's viewpoints", IEEE Transactions on Power Delivery, vol. 29, no. 4, pp. 1831-1840, 2014.

[30] J. Kennedy, and R. Eberhart, "Particle swarm optimization", In: Proceedings of the IEEE International Conference on Neural Networks, Perth, WA, Australia, 1995.

[31] ENWL. Smart Street-Circuit Selection Methodology. July 2014 [Online]. Available: http://www.enwl.co.uk/globalassets/innovation/ smart-street/smart-street-key-docs/smart-street-site-selection-methodol ogy.pdf.

[32] EPRI, Simulation Tool - OpenDSS, [Online] https://smartgrid.epri com/SimulationTool.aspx

[33] CREST Tool, [Online] https://www.lboro.ac.uk/research/crest/demandmodel/

[34] ENWL. Smart Street-Network Design Methodology. July 2014 [Online]. Available: http://www.enwl.co.uk/globalassets/innovation/ smart-street/smart-street-key-docs/smart-street-network-design-method ology.pdf.

[35] J.J. Durillo, A.J. Nebro, C.A. Coello, J. García-Nieto, F. Luna and E. Alba. "A study of multiobjective metaheuristics when solving parameter scalable problems", IEEE Transactions on Evolutionary Computation vol. 14, no. 4, pp. 618-635, 2010.

[36] A. de Campos Jr, A.T. Pozo and E.P. Duarte Jr, "Parallel multi-swarm PSO strategies for solving many objective optimization problems", Journal of Parallel and Distributed Computing, vol. 126, pp. 13-33, 2019.

[37] D. Montenegro, R.C. Dugan and M.J. Reno, "Open Source Tools for High Performance Quasi-Static-Time-Series Simulation Using Parallel Processing", In 2017 IEEE 44th Photovoltaic Specialist Conference (PVSC), pp. 3055-3060, 2017.

[38] Mathworks, MATLAB: Global Optimisation Toolbox, gamultiobj, https://uk.mathworks.com/help/gads/gamultiobj.html

[39] R. Hassan, B. Cohanim, O. De Weck, and G. Venter. "A comparison of particle swarm optimization and the genetic algorithm." In 46th AIAA/ASME/ASCE/AHS/ASC structures, structural dynamics and materials conference, p. 1897, 2005.

[40] D. Divan, W. Brumsickle, R. Schneider, B. Kranz, R. Gascoigne, Ingram, D. M. Bradshaw, and I. Grant, "A distributed static series compensator system for realizing active power flow control on existing power lines", IEEE Transactions on Power Delivery, vol. 22, no. 1, pp. 642-649, 2007.

[41] D. Divan \& H. Johal, (2007) "Distributed FACTS-A New Concept for Realizing Grid Power Flow Control", IEEE Transaction on Power Electronics, vol. 22, pp. 6-11.

[42] H.A. Gabbar, and A.M. Othman, "Performance optimisation for nove green plug-energy economizer in micro-grids based on recent heuristic algorithm", IET Generation, Transmission \& Distribution, vol. 10, no. 3, pp. 678-687, 2016

[43] H. Nafisi, S. M. M. Agah, H. A. Abyaneh, and M. Abedi. "Two-stage optimization method for energy loss minimization in microgrid based on smart power management scheme of PHEVs", IEEE Transactions on Smart Grid, vol. 7, no. 3, pp.1268-1276, 2015.

[44] S.S. Kashyap and Mala De. "Loss allocation and loss minimisation for radial distribution system including DGs", IET Renewable Power Generation, vol. 11, no. 6, pp. 806-818, 2017. 